\title{
The isolation of an X-dependent strain of Haemophilus from otitis media identified as H. haemoglobinophilus (canis)
}

\author{
JOYCE FRAZER AND K. B. ROGERS \\ From the Bacteriology Department, School of Medicine, Leeds, and Department of Microbiology, The \\ Children's Hospital, Birmingham
}

SYNOPSIS The growth factor requirements, together with the serological results, would seem to justify the view that strain M.G., isolated from a child, M.G., with otitis media, in pure culture has to be regarded as $H$. haemoglobinophilus (canis).

It is interesting to note that two out of the three strains of $H$. haemoglobinophilus (canis) available in this laboratory agglutinate to a titre of $1: 80$ with the serum produced against $H$. aphrophilus, the only other X-dependent haemophilus we had used for the preparation of antiserum.

Amongst the many unusual strains of haemophili sent by one of us (K.B.R.) to this laboratory for further investigation was an organism isolated as a pure culture from acute otitis media in a child. This organism subsequently proved to be an X-dependent haemophilus which in all respects was identical to Haemophilus haemoglobinophilus (canis).

M.G. was a girl born on 19 January 1959. She was found to have severe hypogammaglobinaemia and from March 1961 to March 1967 was kept relatively free of chest infections by weekly injections of human gamma globulin: during this period she had many attacks of conjunctivitis, nearly all of which were due to $H$. influenzae, and investigations showed that her tear lysozyme level was significantly below that of normal children: further investigations showed that other patients with hypogammaglobinaemia also had low lysozyme levels in their tears.

Because anaphylactic reactions appeared when the gamma globulin was injected, this therapy had to be stopped and then she unfortunately had several attacks of bronchitis, bronchopneumonia, and otitis media as well as conjunctivitis. Most of these infections were due to $H$. influenzae. In June 1969, at the age of $10 \frac{1}{2}$, she had acute otitis media and from the discharge a pure culture of the organism described here was grown. The sensitivity pattern of this strain suggested to K.B.R. that it might be an unusual haemophilus species different from those previously isolated from this case.

Received for publication 22 July 1971.

\section{Bacteriology}

The routine direct sensitivity plate is made of equal parts of lysed and whole horse blood; on this the haemophilus in the culture of M.G.s ear swab grew as very small colonies. The sensitivities were checked by comparing this strain of haemophilus with a strain of $H$. influenzae isolated from a routine specimen of another patient. The two haemophilus strains were plated on the same chocolate agar sensitivity plate with the antibiotic-containing discs placed in between the two implants. This test confirmed that the M.G. strain had a most unusual pattern of resistance. It was resistant to penicillin, streptomycin, sulphonamides, lincomycin, and cephaloridine, partially sensitive to tetracycline, erythromycin, and ampicillin, and fully sensitive to chloramphenicol, neomycin, polymyxin, and septrin. This was unlike most strains of $H$. influenzae which we isolate, virtually all of which are fully sensitive to streptomycin, tetracycline, erythromycin, ampicillin, and cephaloridine.

The organism grew well in air at $37^{\circ} \mathrm{C}$ and growth was not enhanced by incubation in air with added $\mathrm{CO}_{2}$. On chocolate agar the colonies were small, discrete and clear, becoming opaque after two to three days' incubation. The characteristic 'haemophilus' odour was present. Microscopic examination showed Gram-negative short rods together with a few filaments. Using the method of Zinnemann, Rogers, Frazer, and Boyce (1968) to determine the 
growth factor requirements the organism was consistently X-dependent, and remained so after being freeze-dried for 18 months. Similar to $H$. haemoglobinophilus (canis) nitrate; were reduced to nitrites, the catalase and indole reactions were positive, and oxidase reaction was negative.

Antiserum produced against a standard strain of H. haemoglobinophilus (canis) NCTC 8540 was available because investigations of the serology of several haemophilus species were under way in the laboratory. Strain M.G. agglutinated to full titre against this antiserum.

Absorption of the agglutinating antiserum against strain 8540 with the homologous strain was complete, whilst absorption of the same serum with strain M.G. left a residual titre of 1 in 40 even after reabsorption. It is probable that the residual, unabsorbed antigen is strain specific. This assumption is supported by the result of absorbing serum NCTC 8540 with another $H$. haemoglobinophilus (canis) strain, NCTC 10169, and then testing against the homologous strain. The resultant titre was also 1 in 40 .

Suspensions of strains M.G., H. haemoglobinophilus NCTC 8540 and NCTC 10169, were not agglutinated to titres higher than 1 in 80 by antisera against non-capsulated $H$. influenzae, strain 8405 , a laboratory strain of $\boldsymbol{H}$. parainfluenzae, strain Barts, and $H$. aphrophilus, NCTC 5906 (see Table).

\begin{tabular}{|c|c|c|c|}
\hline \multirow[t]{2}{*}{ Antiserum } & \multicolumn{3}{|l|}{ Strain } \\
\hline & M.G. & 8540 & 10169 \\
\hline H. haemogl. (canis) strain 8540 & $1: 1280$ & $1: 1280$ & $1: 640$ \\
\hline H. influenzae & $1: 10$ & $1: 10$ & $1: 10$ \\
\hline H. parainfluenzae & $1: 20$ & $1: 40$ & $1: 10$ \\
\hline H. aphrophilus & $1: 80$ & $1: 80$ & $1: 40$ \\
\hline
\end{tabular}

Table Agglutination titres of strains M.G. and NCTC nos. 8540 and 10169 to Haemophilus species

The figures are the reciprocal of the agglutination titres.

\section{Discussion}

The growth factor requirements and morphology, together with the serological results, would seem to justify the view that strain M.G. isolated from otitis media in pure culture has to be regarded as H.haemoglobinophilus (canis). It is interesting to note that two out of three strains agglutinated to a titre of
$1: 80$ with the serum produced against $H$.aphrophilus, the only other X-dependent haemophilus species we had used for making an antiserum.

$H$. haemoglobinophilus (canis) was first described by Friedberger in 1903 who isolated it from the prepuce of dogs. He suggested that the organism differed from Pfeiffer's bacillus by showing enhancement of growth in the presence of iron salts. Fleming and Maclean (1930), in the course of identifying strains from the mouth and gums of 20 normal os persons, noted only one X-dependent isolate $\overrightarrow{0}$ amongst 65 strains, which they labelled H. haemo- $\overrightarrow{\vec{H}}$ globinophilus (canis). Isom, Gardy, Selner, Brown, $\vec{\omega}$ and Willis (1964) stated that 'there appears to be no record of $H$. canis infection in man', and we are not aware of any case having been reported since.

It is conceivable that in this case, in one of the $\mathrm{N}$ episodes, $H$. haemoglobinophilus (canis) was the $\vec{v}$ causative microorganism only because the patient's 0 defence mechanism was impaired and that in a normal child such an infection might not have $\vec{T}$ occurred. That this was a single episode was shown $\frac{\mathbb{O}}{0}$ when the patient was readmitted six months later and $H$. parainfluenzae was grown from a throat swab. This species was again isolated three weeks $\vec{\varphi}$ later, and a few days afterwards a strain of $\boldsymbol{H}$. int fluenzae appeared.

The patient has since died but careful questioning of the parents by both the clinician and the health visitor could not uncover continuous close contact with a dog at any time.

We wish to acknowledge the sincere thanks of both of us to Professor K. Zinnemann for unfailing encouragement, advice, and guidance.

References

Fleming, A., and Maclean, I. H. (1930). On the occurrence of influenzae bacilli in the mouths of normal people. Brit. J. exp. Path., 11, 127-134.

Friedberger, E. (1903). Úber ein neues zur Gruppe des Influenzaba-cillus gehöriges hämoglobinophiles Bakterium (Bacillus haemoglobinophilus canis). Zbl. Bact., I. Abt. Orig., 33, 401406.

Isom, J. B., Gardy, P. D., Selner, J. C., Brown, L. J., and Willis (1964). ․ㅡ. Brain abscess due to Haemophilus aphrophilus. New Engl. $\mathrm{N}$ J. Med., 271, 1059-1061.

Zinnemann, K., Rogers, K. B., Frazer, J., and Boyce, J. M. H. (1968). A new V-dependent Haemophilus species preferring increased $\mathrm{CO}_{2}$ tension for growth and named Haemophilus paraphro- $\mathrm{N}$ philus, nov. sp. J. Path. Bact., 96, 413-419. 